\title{
Service-orientation in electronic markets
}

\author{
Rainer Alt • Witold Abramowicz • Haluk Demirkan
}

Published online: 16 November 2010

(C) Institute of Information Management, University of St. Gallen 2010

\begin{abstract}
This special theme aims to analyze the linkage between service-orientation and electronic markets. On the one hand, questions focus on how service-oriented thinking and service-oriented solutions provide value to electronic markets, and on the other how electronic markets improve the effectiveness of service industries, such as healthcare, telecommunications, logistics, education, and others. While the papers in the special theme section highlight selected aspects of a services-based business transformation, this preface discusses main underlying assumptions of the service evolution, underpins the link between serviceorientation and electronic markets, and concludes with some developments on the emerging trans-disciplinary field of service science.
\end{abstract}

\section{Move towards service-orientation}

Service-orientation has emerged as one of the silver bullets in many disciplines such as management, computer science operations, marketing, information systems, and supply chain management. It is substantively grounded in the

\author{
R. Alt $(\square)$ \\ University of Leipzig, \\ Leipzig, Germany \\ e-mail: rainer.alt@uni-leipzig.de \\ W. Abramowicz \\ University of Poznan, \\ Poznan, Poland \\ e-mail: w.abramowicz@kie.ue.poznan.pl \\ H. Demirkan \\ Arizona State University, \\ Tempe, AZ, USA \\ e-mail: haluk.demirkan@asu.edu
}

cross-functional issues of business, engineering, technology and the social sciences. From an economic perspective services are in opposition to physical goods inherently immaterial in nature. While theories from the organizational and marketing science usually capture the nature of these products (e.g. in the area of service-dominant logic), engineering disciplines focus on shaping and developing these information goods (e.g. in the area of service engineering), and the information systems field focuses on integrating services perceived as encapsulated application functionalities with standardized interfaces (e.g. in the area of service-oriented architectures). These research streams also converge in the new interdisciplinary area of service science, which integrates the principles, design, and management of economic as well as technical services. Service science is interested in new forms of value creation using services for organizations, industry structures, consumers, societies and so forth (Chesbrough and Spohrer 2006).

This leads to a vision where businesses or even customers could configure (or co-create) their value chains from services that are provided in application stores or marketplaces which are currently emerging from various vendors. These value chains could be set at multiple scales of organizations, from individual people to businesses and nations, chained together into globally integrated service networks of multiple types: business-to-consumer (B2C), business-to-business (B2B), consumer-to-consumer (C2C), business-to-government (B2G), government-to-consumer/ citizen (G2C), as well as other permutations. Instead of licensing complex standard business software suites, companies would either download the required functionalities (or services) from platforms or use hosted services following the software-as-a-service model (SaaS). Obviously, this may reduce a large portion of the transaction cost 
from initial internal setup overhead to transaction monitoring which is still involved in many of today's business processes. Following the idea of cloud computing, businesses could setup these service-oriented business models with low variable cost settings, which should bring suppliers and customers more efficiently together in an "end-to-end"-vision. While it is even uncertain whether "plugging-and-playing" services among diverse businesses can ever be met due to the many desired heterogeneities in business, we clearly see a variety of enablers evolving in this direction (Bardhan et al. 2010).

First, many companies are increasingly aiming to become more service-oriented in their operations. Following the business reengineering movement that started back in the 1990s, companies analyze their internal services from a customer's perspective and strive for solutions that enable them to combine the flexibility requirements of individualization with the necessities of standardization which are key for efficiency. Another important organizational design element towards an enhanced customer-orientation is the formation of cross-functional service organizations (e.g. shared service or competence centers) that are offering defined services to their internal and/or external customers. Second, the implementation of packaged business software in many companies led to more internal formalization regarding products, processes and data. This also forms a valuable basis for shaping business services that are linked to modular application software. The definition of internal services may be a step to enhance in-sourcing strategies and offer these services on the (external) market. Third, serviceoriented architecture (SOA) principles are spreading in many large organizations as well as with many standard software vendors. To contain the complexity in managing a growing number of IT-based services, platforms comprising services, such as directories, repositories, application or web service marketplaces, are an another enabler. While centralized initiatives, such as Universal Description, Discovery and Integration (UDDI) proved to be viable in the intra-organizational domain only, Web Service intermediaries, such as Strikeiron or RemoteMethods, are only about to emerge.

\section{Service-oriented electronic markets}

Service-orientation and electronic markets are interdependent and closely linked. First of all, electronic markets are intermediaries that are key enablers for reducing the interdependencies among service providers and service consumers. Following the comparison by Kumar and van Dissel (1996), interorganizational systems may feature reciprocal, sequential, and pooled interdependencies. The latter relates to the hub-and-spoke topology which is typical for electronic markets. Due to the standards and rules involved in these settings, these configurations increase the structure and reduce the potential for conflict among the participating actors. Thus, electronic markets are means for loosely-coupling within a service system. Following Spohrer et al. (2007, p. 72) service systems "comprise service providers and service clients working together to coproduce value in complex value chains or networks". From this perspective electronic markets are intermediaries that provide services for the allocation of services as well as the infrastructure for the exchanges or interactions to take place.

A second perspective conceives the electronic markets themselves as service systems. For example, Brohman et al. (2009) describe a network-based customer service system that consists of services for capturing offers, for service discovery and for the generation of proposals. Here, electronic markets are holistic configurations of service systems that permit dynamic value co-creation. Like other service systems, typically electronic markets involve people, technology, organizations, and shared information. Service systems also include such things as language, standards, laws, rules, measures, models, and so on (Spohrer et al. 2007; Demirkan et al. 2009). They are connected internally and externally by value propositions, with governance mechanisms that support and adjudicate the resolution of disputes. Among the services offered by electronic markets are:

(1) Services that support finding, searching, comparing and contracting services. This functional category covers a range of services from the order book and matching mechanisms typically to be found in the area of financial as well as other commodity exchanges, from the catalog services of electronic markets in the area of tourism as well as procurement, to the directory services (e.g. service repositories and registries) that are an important architectural element in managing the complexity of services in distributed computing concepts (e.g. SOA). Standards that capture business knowledge, such as the Unified Service Description Language (USDL) are increasingly emerging for the necessary semantics needed when describing and cataloguing services.

(2) Services that enable the execution of processes via the electronic market platform. For example, established electronic markets in the financial sector have sophisticated procedures in place for settling and clearing orders. Other electronic markets, such as the catalog markets mentioned above, provide services for billing and payment, order fulfillment as well as logistics. As this category refers to services on a process level, it also includes services for the orchestration of individual services to become service chains. Technologically speaking, an existing standard that supports the binding of services is the Business Process Execution Language (BPEL) that executes services that were defined with the Business Process Modeling Notation (BPMN). 
(3) A third group of services refers to the infrastructure provided by electronic markets. In financial markets this would apply to surveillance services, analytical services and trading partner registries. Other markets comprise trust services that ensure a secure transaction environment and mapping services for messaging purposes on a technical level. Among the standards in this category are the SOAP protocol in the Web Services domain and standards for data syntax, such as the FIX protocol or EDIFACT.

As shown in Fig. 1, a service-oriented perspective on electronic markets not only substantiates the distinction of electronic markets as part of a value chain service system and as separate service systems, but also theories, principles, methodologies, and tools for the engineering, management, delivery and implementation of these service systems. In particular, electronic market providers could use service science to define and to manage their own service portfolio as well as the services from external service providers. The explicit notion of the interaction process recognizes that specified services are exchanged following certain interaction principles and procedures, such as co-creation and service level agreements. The vision introduced above which sees the flexible choreography and orchestration of business services within a single and even among multiple value chain service systems, would require that all service perspectives mentioned need to be addressed.

\section{Papers in this special theme}

Clearly, this broad scope of future research is beyond the contribution of this special theme. However, the framework is helpful in positioning the three research papers in a larger context. While the first focuses on the role of technological architectures for the networking among businesses, the second analyzes the virtualization of services which may be used via electronic markets, and the third the service quality of electronic markets. All papers underwent a minimum of two revisions and we highly appreciate the dedication of all reviewers that made this special theme possible.
The first contribution by Jan Löhe and Christine Legner titled "SOA Adoption in Business Networks" raises the question whether service-oriented architectures really advance interorganizational integration. It starts from the observation that SOA is spreading in practice and that SOA is attributed with a variety of potentials that might influence the formation of business networks. Indeed, the authors show that SOA fosters the participation in business networks as well as endto-end-integration and more complex electronic interactions. The paper links established research on interorganizational systems (IOS) with current research on SOA and introduces a multi-dimensional classification scheme which is used to formulate five clusters of SOA adoption in a value chain service system.

The second contribution from Christian Czarnecki, Axel Winkelmann and Myra Spiliopoulou is titled "Services in Electronic Telecommunication Markets" and presents an approach on how process components may be virtualized using a conceptual framework. It shows that most of the telecommunication companies neither take advantage of the SOA paradigm nor use software components offered by partners or available in the Web while delivering a service to a client. Instead, they prefer to have their business processes (and therefore also services) tightly coupled with their own infrastructure. They perceive process virtualization as one of the major risks for their business affecting the quality of service offered to customers. From this starting point the authors see customer-orientation as a basis for the prioritization of processes to be virtualized. In the article, they use a customer-centric perspective to undertake the task of process virtualization and propose guidelines for removing internal virtualization barriers. They also determine factors that affect the 'virtualizability' of processes, using the underpinnings of the Process Virtualization Theory (PVT) proposed by Overby. The article concludes with a case study validating the proposed approach.

Finally, the third contribution authored by Shariar Akter, John D'Ambra and Pradeep Ray analyzes the service quality of mHealth Platforms using a hierarchical model based on the partial least squares method. Following this research the service quality of platforms, such as electronic markets, has multiple dimensions which include platform quality, interaction quality, and outcome quality. This
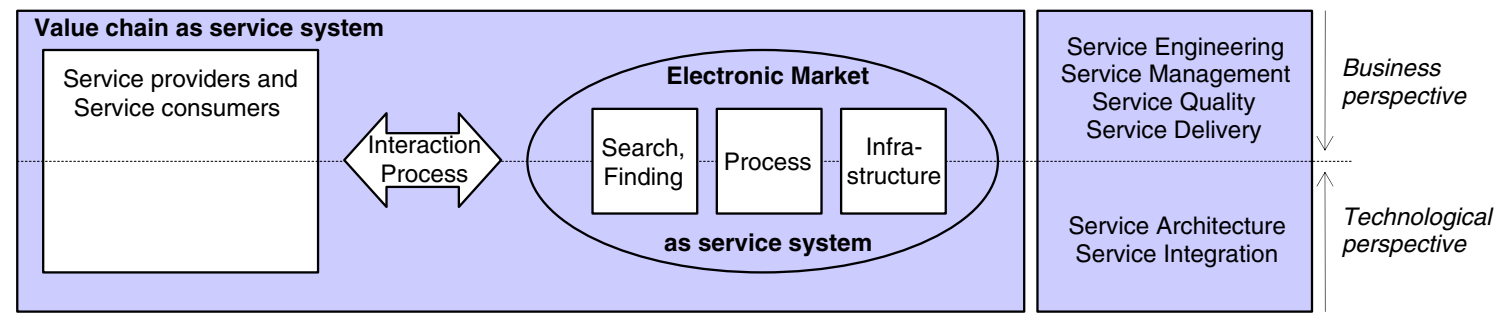

Fig. 1 Facets of service-orientation on electronic markets 
measurement introduces novel constructs, such as the intention to continue using the platform (ICU) and the quality of life (QOL), which were also empirically tested with telemedicine customers in Bangladesh. Among the findings are that service satisfaction has an important mediating role on the both ICU as well as QOL and promises a helpful contribution for platform providers when analyzing and engineering their service delivery systems.

\section{Conclusion}

To summarize, electronic markets are positioned for its next generation as thousands of customers and companies are connected to each other with the fast growing Internet. In the future instead of interacting with "do-it-yourself" web store fronts, customers will be able to communicate (e.g. search, buy, sell) with the businesses via automated service-oriented market models. Businesses will be able to co-develop and provide highly customized products and services according to customers' preferences along value chain service systems. Also, businesses will be able to reduce the cost of services by using commoditized business processes, architecture and infrastructure that is as a result of service-orientation. Of course, technological advancements will not slow down and the mobile technologies will enable customers to buy in anywhere and anytime. Of course, these networked and highly customized service offerings will use large amounts of data and information. Of course, services that are either provided or requested by individual companies via electronic markets would require a considerable amount of standardization and semantics which would also apply to the categories and structures in the electronic markets service system. Of course, many efforts are still necessary to make next generation electronic markets a reality, but at the same time open a broad spectrum of challenges and opportunities for service science researchers and practitioners.

\section{References}

Bardhan, I. R., Demirkan, H., Kannan, P. K., Kauffman, R. J., \& Sougstad, R. (2010). An interdisciplinary perspective on services management and service science. Journal of Management Information Systems, 26(4), 13-64.

Brohman, M. K., Piccoli, G., Martin, P., Zulkernine, F., Parasuraman, A., \& Watson, R. T. (2009). A design theory approach to building strategic network-based customer service systems. Decision Sciences, 40(3), 403-430.

Chesbrough, H., \& Spohrer, J. (2006). A research manifesto for services science. Communications of the ACM, 49(7), 35-40.

Demirkan, H., Kauffman, R. J., Vayghan, J. A., Fill, H.-G., Karagiannis, D., \& Maglio, P. P. (2009). Service-oriented technology and management: perspectives on research and practice for the coming decade. The Electronic Commerce Research and Applications Journal, 7(4), 356-376.

Kumar, K., \& van Dissel, H. G. (1996). Sustainable collaboration: managing conflict and cooperation in interorganizational systems. MIS Quarterly, 20(3), 279-300.

Spohrer, J., Maglio, P. P., Bailey, J., \& Gruhl, D. (2007). Steps toward a science of service systems. IEEE Computer, 40(1), 71-78. 